\title{
HIV-1 Drug Resistance and Genetic Diversity among Vertically Infected Cameroonian Children and Adolescents
}

\author{
Béatrice Dambaya ${ }^{1,2 \#}$, Joseph Fokam ${ }^{1,3,4 \# *}$, Ezéchiel Semengue Ngoufack ${ }^{1,5,6}$, Désiré Takou ${ }^{1}$, \\ Maria Mercedes Santoro ${ }^{6}$, Georges Této ${ }^{1}$, Grâce Angong Beloumou ${ }^{1}$, \\ Linda Chapdeleine Mekue Mouafo ${ }^{1,7}$, Nelly Kamgaing ${ }^{1,3,8}$, Samuel Martin Sosso ${ }^{1}$, \\ Serges Clotaire Billong ${ }^{3,4,9}$, Anne Esther Njom Nlend ${ }^{10,11}$, Martin Sanou Sobze ${ }^{5}$, \\ Céline Nkenfou ${ }^{1,12}$, Paul Ndombo Koki ${ }^{2,13}$, Flobert Njiokou ${ }^{2}$, Vittorio Colizzi ${ }^{1,6}$, \\ Carlo Federico Perno ${ }^{1,14}$ and Alexis Ndjolo ${ }^{1,2}$
}

\begin{abstract}
${ }^{1}$ Chantal BIYA International Reference Centre for research on HIV/AIDS prevention and management (CIRCB), Yaounde, Cameroon; ${ }^{2}$ Faculty of Sciences, University of Yaounde I, Yaounde, Cameroon; ${ }^{3}$ Faculty of Medicine and biomedical Sciences, University of Yaounde I, Yaounde, Cameroon; ${ }^{4}$ National HIV Drug Resistance working Group, Ministry of Public Health, Yaounde, Cameroon; ${ }^{5}$ Faculty of Medicine and Pharmaceutical Sciences, University of Dschang, Dschang, Cameroon; ${ }^{6}$ University of Rome Tor Vergata, Rome, Italy; ${ }^{7}$ Faculty of Sciences,

University of Dschang, Dschang, Cameroon; ${ }^{8}$ University Health Center, Yaounde, Cameroon; ${ }^{9}$ Central Technical Group, National AIDS Control Committee, Yaounde, Cameroon; ${ }^{10}$ National Social Welfare Hospital, Yaounde, Cameroon; ${ }^{11}$ Higher Institute of Medical Technology, University of Douala, Douala, Cameroon; ${ }^{12}$ Higher Teacher Teaching College, University of Yaounde I, Yaounde, Cameroon; ${ }^{13}$ Mother-Child Centre, Chantal BIYA Foundation, Yaounde, Cameroon; ${ }^{14}$ University of Milan, Milan, Italy; ${ }^{7}$ These authors contributed equally to this work.
\end{abstract}

\begin{abstract}
Background and objective: HIV-1 vertically infected children stand a high risk of HIV-1 drug resistance (HIVDR), especially after failure to prevention of mother to child transmission (PMTCT) and pediatric antiretroviral therapy (ART). Thus, surveillance of HIVDR might contribute in delineating optimal pediatric regimens. The objective of this study was to evaluate HIVDR and subtype distribution among ART-naïve and ART-failing children.
\end{abstract}

Methods: A study was conducted throughout 2017 amongst 102 children/adolescents at the "Chantal BIYA International Reference Centre" (CIRCB) in Cameroon. HIVDR testing was performed in protease-reverse transcriptase (RT) region and interpreted using the Stanford HIVdbv8.5; subtyping was performed using MEGA v7.0.26; and data were analyzed using Epi-info v7.1.3.3, with $p<0.05$ considered statistically significant.

Results: Sequences were generated from 63 participants (19 ART-naïve, 44 ART-failure); the median-age was respectively 6 [IQR:3.5-11] and 144 [IQR:116.25-185]

Keywords: Drug resistance; HIV subtypes; Children; Adolescents; Cameroon. Abbreviations: ADR, acquired HIV drug resistance; ART, antiretroviral therapy; CRFs, circulating recombinant forms; DR, drug resistance; DRMs, drug resistance mutations; DRV/r, ritonavir boosted darunavir; EID, early infant diagnosis; GRT, genotypic HIV-1 drug resistance testing; HAART, highly active ART; HIVDR, HIV1 drug resistance; IQR, interquartile range; NNRTI, non-nucleoside RT inhibitors; NRTI, nucleoside RT inhibitors; PDR, pre-treatment HIV drug resistance; PMTCT, prevention of mother to child transmission; PI, protease inhibitors; SSA, sub-Saharan Africa; sd-NVP, single dose nevirapine; WCA, West and Central Africa; WHO, World Health Organization.

Received: December 06, 2019; Revised: January 10, 2020; Accepted: January 19, 2020 *Correspondence to: Joseph Fokam, Chantal BIYA International Reference Centre for research on HIV/AIDS prevention and management (CIRCB); Faculty of Medicine and biomedical Sciences, University of Yaounde I; National HIV Drug Resistance working Group, Ministry of Public Health, Yaounde, Cameroon. Tel: +237222315450; E-mail: fokamjoseph@circb.cm, josephfokam@gmail.com

How to cite this article: Dambaya B, Fokam J, Ngoufack ES, Takou D, Santoro MM, Této G, Beloumou GA, Mouafo LCM, Kamgaing N, Sosso SM, Billong SC, Njom Nlend AE, Sobze MS, Nkenfou C, Koki PN, Njiokou F, Colizzi V, Perno CF, Ndjolo A. HIV-1 Drug Resistance and Genetic Diversity among Vertically Infected Cameroonian Children and Adolescents. Exploratory Research and Hypothesis in Medicine 2020;5(2):53-61. doi: 10.14218/ERHM.2019.00031. months for ART-naïve and ART-failing (median ARTduration: 23.55 [IQR:7.61-60.91] months, 63.6\% receiving non-nucleoside RT inhibitors [NNRTI]-based regimens). Among ART-naïve children, overall-HIVDR was $52.6 \%$ (10/19), with $31.6 \%$ (6/19) to NNRTI, $26.3 \%$ (5/19) to nucleoside RT inhibitors (NRTI) and $15.8 \%$ (3/19) to ritonavir-boosted protease inhibitor (PI/r). Among ART-failing children, overall-HIVDR was $97.7 \%$ (43/44), with 95.4\% (42/44) to NNRTI, 90.9\% (40/44) to NRTI and $18.2 \%(8 / 44)$ to PI/r. Multi-drug resistance was found in $21.05 \%$ (4/19) ART-naïve versus $85.7 \%$ (24/28) on NNRTI-based and 50\% (8/16) on PI-based regimens; $O R=4.36, p=0.045$. CRF02_AG was prevalent $(68.2 \%)$, without any effect on HIVDR $(p=0.99)$.

Conclusions: The high rates of HIVDR, in both ART-na- 
ïve and ART-failing children, suggest using genotypic HIV-1 drug resistance testing for selecting optimal pediatric ART-regimens. Multi-drug resistance is concerning among children failing ART and prompts the need of new drugs (integrase inhibitors, darunavir/ritonavir) for optimal pediatric ART management.

\section{Introduction}

In spite of the $70 \%$ decline in the overall incidence of pediatric HIV infections between 2000 and 2015, an estimated 180.000 children became infected worldwide in 2017, giving a total of 1.8 million children $(<15$ years old) living with HIV globally, of whom $90 \%$ live in sub-Saharan Africa (SSA) ${ }^{1-3}$ Even though there is progress in the universal coverage of pediatric antiretroviral therapy (ART) in SSA, risks of pediatric HIV-associated mortality remain concerning in this setting due to, but not limited to, delayed initiation and suboptimal monitoring of ART, added to the known high viral load, the immunological immaturity at early age and rapid disease progression among infected children. ${ }^{4-6}$

Though progress in the HIV prevention of mother to child transmission (PMTCT) services, ranging from options A (i.e. azidothymidine $[\mathrm{AZT}]$ ), $\mathrm{B}$ (i.e. ART during pregnancy and breastfeeding) to the current $\mathrm{B}+$ (i.e. lifelong ART regardless of clinical and immunological status), represents the cornerstone in eliminating new cases of pediatric infections, ${ }^{1,7,8}$ rates of HIV vertical transmission are still beyond five percent in several PMTCT high priority countries, including Cameroon. ${ }^{4-7}$ Furthermore, risks of both pretreatment and acquired HIV drug resistance (HIVDR) become more threatening for every infected child in the frame of failure to current PMTCT strategy and pediatric ART services respectively. ${ }^{8-10}$ Of note, one in two (50\%) infected children harbored pretreatment HIVDR and treated children experience early ART failure with acquired HIVDR. ${ }^{10}$ Thus, pediatric HIVDR may represent a major hurdle in achieving the third pillar of the 90-90-90 targets in children. ${ }^{10}$ This hypothesis is plausible in West and Central Africa (WCA) where a total of 2.4 million people, including PMTCTattendees, are receiving ART (i.e. 40\% [25-55\%] coverage) and the overall viral suppression rate is still around $70 \%$, with lower outcomes in the pediatric populations. ${ }^{6,10}$

As in several WCA countries, the therapeutic management of pediatric HIV in Cameroon follows guidelines from the World Health Organization (WHO). ${ }^{11}$ Of note, initial (first-line) ART depends on PMTCT-exposure: either (a) ritonavir-boosted protease-inhibitor (PI)-based for PMTCT-exposed HIV-infected children below three years of age, or (b) non-nucleoside reversetranscriptase inhibitor (NNRTI)-based regimens for peers without PMTCT-exposure and for children aged three years and above (regardless of PMTCT-exposure). In the advent of ART failure (two consecutive viral loads of at least 1,000 RNA copies $/ \mathrm{mL}$ ), therapeutic ART switch is recommended without referring to genotyping for optimal ART selection. ${ }^{8-10,12-14}$ Thus, in a context with evolving PMTCT interventions, poor adherence to ART and limited access to ART monitoring, suboptimal therapeutic response might be unwavering in sustaining the risks of HIVDR among children initiating or failing ART. ${ }^{15,16}$

In Cameroon, the rate of HIV MTCT is still beyond $5 \%{ }^{17}$; ART coverage in children is still below $50 \%{ }^{18}$; viral suppression is around $60-80 \%$ for children and slightly above $50 \%$ for adolescents. ${ }^{19,20}$ Prior to wide scale-up of PMTCT, pretreatment HIVDR was low (4.9\%) while acquired HIVDR was very high (90\%) following ART failure at a median of two years in this country. ${ }^{21,22}$ After the wide coverage of PMTCT, the increasing access to ART for children and adolescents and the fast growing genetic diversity of HIV-1 in the country, it is then crucial to set-up bold and innovative approaches in the pediatric AIDS response tailored to the specific needs of the local epidemic. ${ }^{23}$

With the goal to generate new findings, in the era of option $\mathrm{B}+$, towards informed decision-making for more efficient ART strategies and therapeutic monitoring in children, we sought to determine the rates of pre-treatment HIVDR (PDR), acquired HIVDR (ADR) and the HIV-genetic variability among vertically infected Cameroonian children.

\section{Materials and methods}

\section{Study design, settings and population}

A cross-sectional and analytical study was conducted throughout the year 2017 in a population of 102 HIV-infected children and adolescents (33 ART-naïve and 69 ART-experienced) from four regions of Cameroon: Center, Littoral, West and North-West regions.

Laboratory analyses for HIV-1 early-infant diagnosis (EID), viral load measurement, CD4 T cells enumeration and HIVDR testing were carried out at the Chantal BIYA International Reference Centre for HIV/AIDS prevention and management (CIRCB), located in Yaoundé, Cameroon.

The CIRCB is a government institution of the Ministry of Public Health dedicated to HIV research and patient monitoring in several aspects, among which: (a) HIV early infant diagnosis in the frame of the national PMTCT program; (b) diagnosis of co-infections with HIV; (c) viral load measurement; (d) CD4 and CD8 T lymphocytes counts; (e) biochemical and hematological tests for drug safety; (f) genotypic HIVDR testing (GRT) at subsidized costs; with quality control programs conducted in partnership with Quality Assessment and Standardization of Indicators (QASI) and other international agencies (http://www.circb.cm/btc_circb/web/).

\section{Ethical considerations}

Administrative authorizations were issued by the CIRCB and the Ministry of Public Health ( $\left.{ }^{\circ} 2014 / 05 / 631\right)$; ethical clearance for the study was obtained from the National Ethics Committee for research on human health (Authorization Number: N²017/03/877/ $\mathrm{CE} / \mathrm{CNERSH} / \mathrm{SP}$ ); written informed consent for each participant was obtained from parents or legal guardians; data were protected by the use of specific identifiers for purpose of confidentiality and stored in a password encrypted computer; all laboratory results were freely returned to participants for benefit in their personal clinical management.

\section{Enrolment of study participants}

On one hand, infants from the aforementioned clinical sites, referred to CIRCB for HIV-1 EID and whose result was HIV positive, were included in the PDR study arm. On the other hand, ARTexperienced children, with a suspicion of ART failure from one of the clinical sites, were also referred to CIRCB for GRT in case of a 
confirmed virological failure (VF).

\section{CD4 cell count and viral load measurement}

CD4 cell count was performed using the BD FACS Count system as per the manufacturer's instructions (https://www.bdbiosciences. com/documents/BD_FACSCount_Brochure.pdf).

HIV-1 RNA quantification was performed on plasma samples using the Abbott m2000rt Real Time HIV-platform (Abbott Molecular Inc. 1300 E. Touhy Ave. Des Plaines, IL 60018 200680-105; USA) according to manufacturer instructions (www.abbottmolecular.com/products/infectious-diseases/realtime-pcr/hiv-1-assay). Briefly, a protocol using $0.6 \mathrm{~mL}$ of plasma was used for RNA extraction, followed by a simultaneous amplification and detection on a real-time polymerase chain reaction (RT-PCR). The lower and upper detection threshold of the assay was respectively $<40$ and $>10.000$ HIV-1 RNA copies/mL.

\section{HIV-1 genotypic drug resistance testing}

HIV-1 GRT was performed on plasma samples following an inhouse protease-reverse transcriptase genotyping assay. ${ }^{24}$ Briefly, RNA was extracted after concentration from $1 \mathrm{~mL}$ of plasma aliquots using the QIAamp Viral RNA mini kit (Qiagen, Milan, Italy), according to the manufacturer's protocol. RNA was then retrotranscribed and amplified using the kit One-Step Invitrogen (Foster City, CA) (SuperScript One-Step for long templates RTPCR) and 2 different sequence-specific primers [ $« 5^{\prime}$-GAC AGG CTA ATT TTT TAG GG-3'» (2075-2094 pol) and « '5' -GAT AAA TTT GAT ATG TCC ATT G-3'» (3555-3576 pol)] for 40 cycles. A second round PCR (semi-nested PCR) was performed with another set of two primers for 35 cycles [ $« 5^{\prime}$ - GAC AGG CTA ATT TTT TAG GG- 3'» $(2075-2094 \mathrm{pol})$ and $« 5^{\prime}-\mathrm{CCT}$ TGT TTC TGT ATT TCT GCT $-3^{\prime} \gg(3527-3547$ pol)]. After PCR products purification using Amicon kits, a direct sanger-sequencing reaction was then performed using 8 overlapping primers [ $« 5^{\prime}$-AGC AGA CCA GAG CCA ACA GC-3'» (2140-2159 gag), «5'-CCA TCC ATT CCT GGC TTT AAT-3'» (2582-2602 pol), «5'-CAG GAA TGG ATG GCC CAA AA-3'» (2590-2609 pol), «5'-TTG TAC AGA AAT GGA AAA GGA AGG-3'» (2660-2683 pol), «5'-CCC TGT GGA AAG CAC ATT GTA-3'» (2985-3004, with an insertion), «5'-GCT TCC ACA GGG ATG GAA A-3'» (2993-3011 pol), «5'-CTA TTA AGT CTT TTG ATG GGT CA-3'» (3506-3528 pol) «5'-CCT TGT TTC TGT ATT TCT GCT-3'» (3527-3547 pol)] and the final product was purified using Sephadex G-50 fine powder. ${ }^{24}$

\section{HIV-1 sequence analysis}

Sequence data were obtained after capillary electrophoresis on a Genetic Analyzer (Applied Biosystems 3500; Life Sciences, Foster City, CA; 08 capillaries), and complete sequences encompassing the pol region of interest ( 1,297 nucleotides) and corresponding to the entire protease region and the first 300 amino acids of the reverse transcriptase open reading frame) were assembled and manually edited using Seqscape software v2.7 (Applied Biosystems, Foster city, CA).

\section{HIV drug resistance mutations and interpretation}

Sequences generated were analyzed for DR mutations (DRMs) us- ing the Stanford University HIV Drug Resistance Database (http:// www.hivdb.stanford.edu) and reported separately for ART-naïve and ART-failing children. In each study group, frequency and types of HIVDR mutations were reported. Sequences having a mixture of wild-type and mutant residues at single drug-resistance-associated positions were also considered to have the mutants at those positions. The nucleotide sequences from this study are submitted to a public repository under the following accession numbers: GenBank MK867695-MK867757.

\section{Phylogeny analysis}

All the generated sequences were aligned in BioEdit version 7.2.6 (Tom Hall, Raleigh, NC) using CLUSTAL W, and compared with reference sequences for the major HIV-1 subtypes and circulating recombinant forms (CRFs), available in the Los Alamos database (http://www.hiv.lanl.gov), gaps were then removed from the final alignment. The phylogenetic tree was inferred using Maximum likelihood method on the MEGA software v7.0.26 for both subtyping and to ensure that there was no cross-contamination of samples. The statistical robustness and reliability of the branching order within the phylogenetic tree were confirmed through a bootstrap analysis using 1,000 replicates on a maximum likelihood tree obtained by molecular phylogeny. Recombination among HIV-1 clades was assessed by HIV BLAST (https:/www.hiv.lanl.gov/ content/sequence/BASIC_BLAST/basic_blast.html), REGA v3 (REGA Institute, KU Leuven, Belgium), COMET (https://comet. lih.lu/index.php?cat=hiv1), RDP $4^{25}$ (Oxford, England) and SplitTree4 v4.14.6 (Tuebingen University, Germany; Otago University, New Zealand).

\section{Statistical analysis}

Data were analyzed using the Epi-info v7.1.3.3, Microsoft Access and Excel 2013. Parametric and non-parametric tests were used for both univariate and multivariate analysis. Fisher exact test, Chi square test and Spearman correlation were used to describe the associations between our variables with $\mathrm{p}$-values $<0.05$ considered statistically significant.

\section{Results}

\section{Sequencing performance and study enrollees}

Out of the 102 children/adolescents enrolled in the study, lowlevel viremia ( $<3$ Log RNA copies $/ \mathrm{mL}$ ) was found from $33 \mathrm{sam}-$ ples, likely due to either prophylactic effect of PMTCT-exposure $(n=13)$ or the therapeutic effect of ART $(n=20)$. Of the 69 patients with eligible viremia for sequencing ( $\geq 3$ Log RNA copies/ $\mathrm{mL}), 63$ completed sequences were generated, giving a sequencing performance of $91.3 \%$, as shown in the flow chart (Fig. 1). Thus, only the 63 patients with available sequences (accession numbers MK867695-MK867757) were included as study participants in the final dataset for analysis.

\section{Characteristics of study participants}

Of the 63 study participants considered for analysis, the sex distribution was similar $(52.4 \%$ boys and $47.6 \%$ girls $)$ and their age 


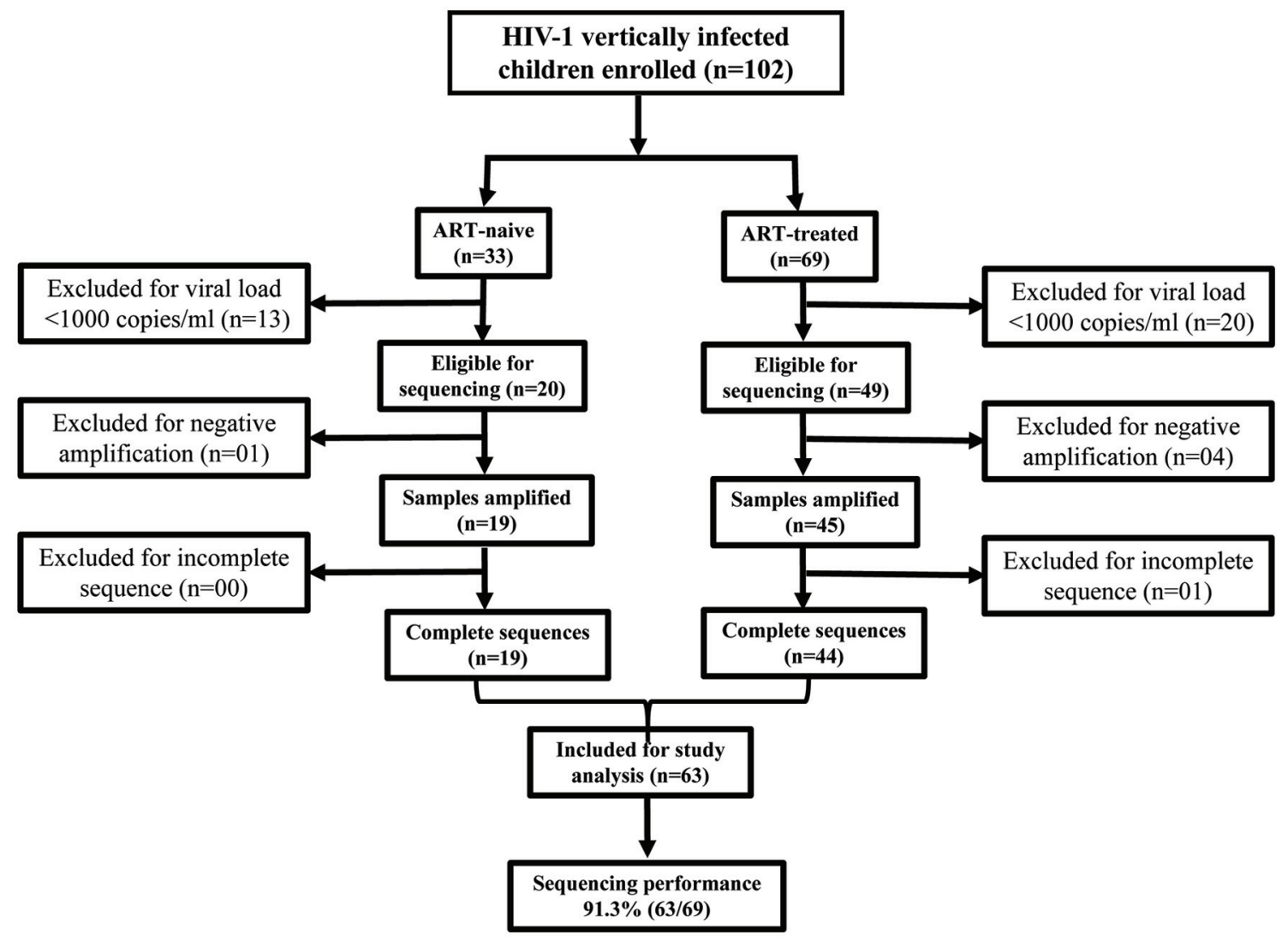

Fig. 1. Flow chart of enrolment and sequencing performance. ART, antiretroviral therapy.

ranged from 2 to 239 months. According to exposure to highly active ART (HAART), 19 children were naïve to HAART and 44 were experiencing VF following exposure to HAART.

Among HAART-naïve participants $(\mathrm{n}=19)$, the median age [interquartile range, IQR] was 6 [3.5-11] months, the CD4 count ranged from $12-2,897$ cells $/ \mathrm{mm}^{3}$ and the viral load ranged from 3,855-10,000,000 HIV-1 RNA copies/mL. According to PMTCT prophylaxis, seven were reported not to have being exposed to antiretrovirals (ARV), while 12 were reported exposed to ARV prophylaxis of which nine exposed to single dose nevirapine (sdNVP), two exposed to AZT and one exposed to both NVP and AZT (see Table 1).

Among participants on ART experiencing VF, the median age [IQR] was 144 [116.25-185] months; CD4 count ranged from $2-3,436$ cells $/ \mathrm{mm}^{3}$ and the plasma viral load from $1,280-$ $6,664,000$ copies $/ \mathrm{mL}$. The median duration on ART [IQR] was $23.55[7.61-60.91]$ months, with $63.6 \%(28 / 44)$ receiving an NNRTI-based regimen versus $36.4 \%$ (16/44) receiving a PI/r-based regimen.

\section{Pre-treatment HIV-1 drug resistance in the study population}

Among HAART-naïve participants, the overall threshold of PDR was 52.6\% (10/19). Considering reports of PMTCT-exposure, $50 \%(6 / 12)$ of participants reported to be exposed to ARV for PMTCT prophylaxis harbored PDR mutations while 57.14\% (4/7) of those reported without any ARV exposure harbored PDR; without any statistically significant difference between the two groups $(\mathrm{p}=0.57)$

The rate of PDR varied by ARV drug class, ranging from $31.6 \%$ (6/19), 26.3\% (5/19) and 15.8\% (3/19) resistance to NNRTI, NRTI to $\mathrm{PI} / \mathrm{r}$ respectively; which were all far above the WHO's threshold of $10 \%$ PDR (Fig. 2). Interestingly, multi-drug resistance was observed in $21.05 \%(4 / 19)$ of participants: two being resistant to both NRTI and NNRTI, one to both NRTI and PI/r, and one to both NNRTI and PI/r.

Of the six participants harboring major NNRTI-DRMs, mutation Y181C was the most frequent (5/6) in RT-region, conferring high levels of resistance to both first and second generation NNRTIs. Of the five participants with major NRTI-DRMs, L74V/I was the most frequent $(2 / 5)$ in the RT-region, conferring resistance to abacavir $(\mathrm{ABC})$ and in lesser extend to tenofovir (TDF). Of the three participants with PI/r-DRMs, equal frequencies $(1 / 3)$ of the mutations M46I, V32I, and D30N were found in the PR-region, conferring intermediate levels of resistance to ritonavir boosted lopinavir, ritonavir boosted atazanavir and even ritonavir boosted darunavir (DRV/r) to certain extent.

\section{Acquired HIV drug resistance in the study population}

Among participants experiencing VF, the overall rate of ADR was $97.7 \%$ (43/44), stratified by drug class as follows: $95.4 \%(42 / 44)$, $90.9 \%(40 / 44)$ and $18.2 \%(8 / 44)$ resistance respectively to $\mathrm{NN}$ RTI, NRTI and PI/r. Regarding multi-drug resistance, dual-class of ADR (NNRTI and NRTI) was $85.71 \%$ (24/28) versus $93.75 \%$ $(15 / 16)$ among those on first-line versus second-line ART ( $p=$ 
Table 1. Description of PMTCT prophylaxis in HAART-naïve population

\begin{tabular}{lll}
\hline Age of the child (in months) & ARV prophylaxis & Duration of prophylaxis \\
\hline 2 & Sd-NVP & 6 weeks \\
2 & Sd-NVP & 6 weeks \\
3 & Sd-NVP & 6 weeks \\
4 & Sd-NVP & 6 weeks (as from week 3) \\
5 & AZT & 6 weeks \\
6 & Sd-NVP & 6 weeks \\
7 & Sd-NVP and then AZT & 6 weeks \\
8 & Sd-NVP & 6 weeks \\
8 & Sd-NVP & 6 weeks \\
9 & AZT & 6 weeks \\
26 & Sd-NVP & 6 weeks (as from month 3) \\
169 & Sd-NVP & 6 weeks \\
\hline
\end{tabular}

ARV, antiretroviral; AZT, azidothymidine; HAART, highly active antiretroviral therapy; PMTCT, prevention of mother-to-child transmission of HIV; Sd-NVP, single dose nevirapine.

0.638); while triple class of ADR (NNRTI, NRTI and PI/r) was $0 \%$ $(0 / 28)$ versus $50 \%(8 / 16)$ respectively among those on first-line versus second-line ART $(\mathrm{p}<0.0001)$.

Among the 42 participants harboring NNRTI-DRMs, K103NS (19/42) and Y181C (16/42) mutations were the most frequent; among the 40 participants harboring NRTI-DRMs, M184VI was the most frequent (34/40), followed by Thymidine Analogue $\mathrm{Mu}-$ tations (T215YF [25/40], and M41L [18/40]); among the eight participants with PI/r-DRMs, M46IV (6/8), V82A (3/8) and L76V $(3 / 8)$ mutations were the most frequent.

\section{Potential efficacy of antiretroviral drugs for clinical management}

According to ART-regimens and based on the genotypic susceptibility scores, the predictive efficacies of drug class ranged from
$57.89 \%$ for NNRTIs to $89.47 \%$ for PI/r among HAART-naïve participants; from $0.00 \%$ for NNRTIs to $100.00 \%$ for PI/r among those failing first-line ART; and from $0.00 \%$ for NNRTIs to $50.00 \%$ for $\mathrm{PI} / \mathrm{r}$ among those failing second-line ART (Fig. 3). Of note, only those failing second-line had a considerable reduced activity of $\mathrm{PI} / \mathrm{r}(50 \%)$, with about $18.75 \%$ (3/16) showing a reduced activity of DRV/r.

\section{HIV-1 genetic diversity in the study population}

In the entire study population, 13 different viral strains were found (seven in the populations of HAART-naive and eight in the population of ART-failure), with a prevailing circulating recombinant form (CRF), CRF02_AG (68.2\%), followed by G (9.5\%), F2 $(4.8 \%)$ and other subtypes (17.5\%), as shown in Figure 4.

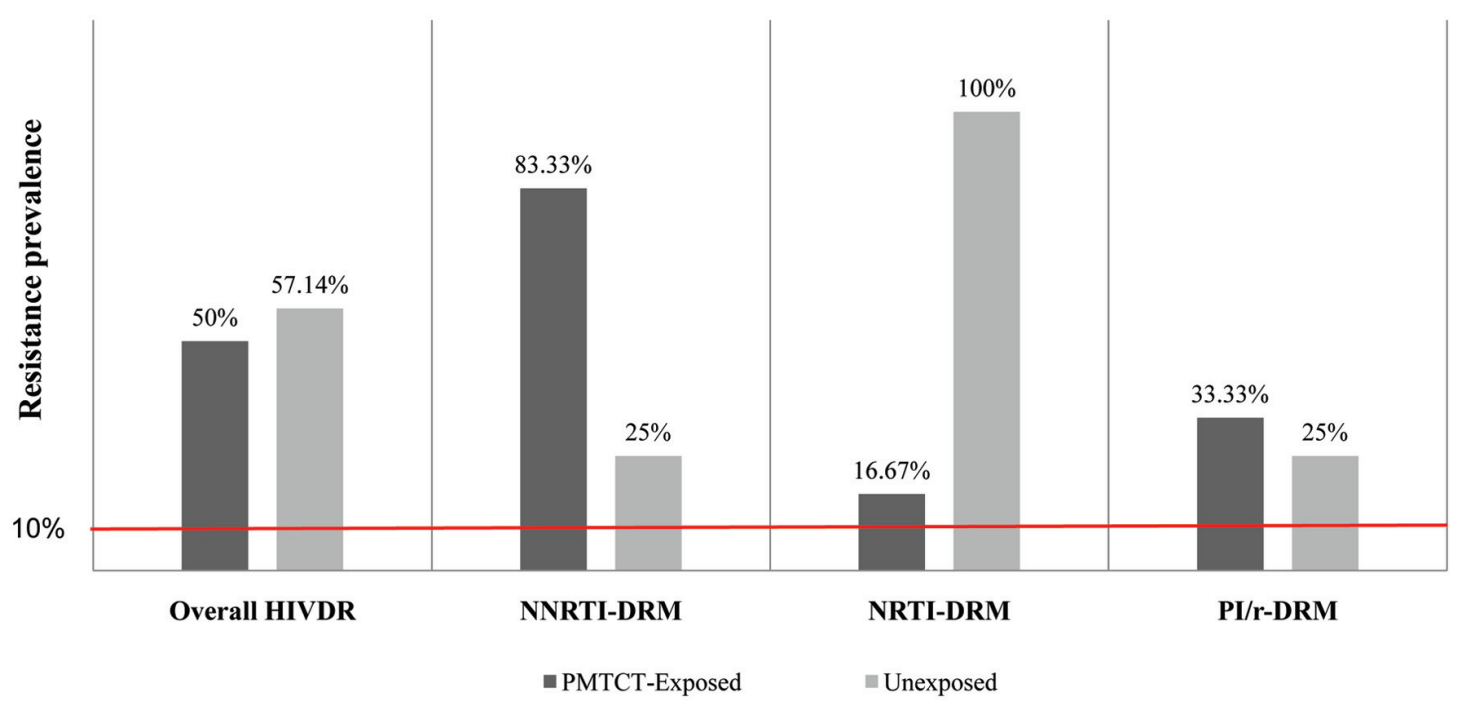

Fig. 2. Distribution of pre-treatment HIV drug resistance according to PMTCT-exposure. Horizontal line at $10 \%$ indicates the threshold established by the World Health Organization to consider high rate of pre-treatment drug resistance. HIVDR, HIV drug resistance; NNRTI, non-nucleoside reverse transcriptase inhibitors; DRM, drug resistance mutation; $\mathrm{Pl} / \mathrm{r}$, ritonavir-boosted protease inhibitor. 


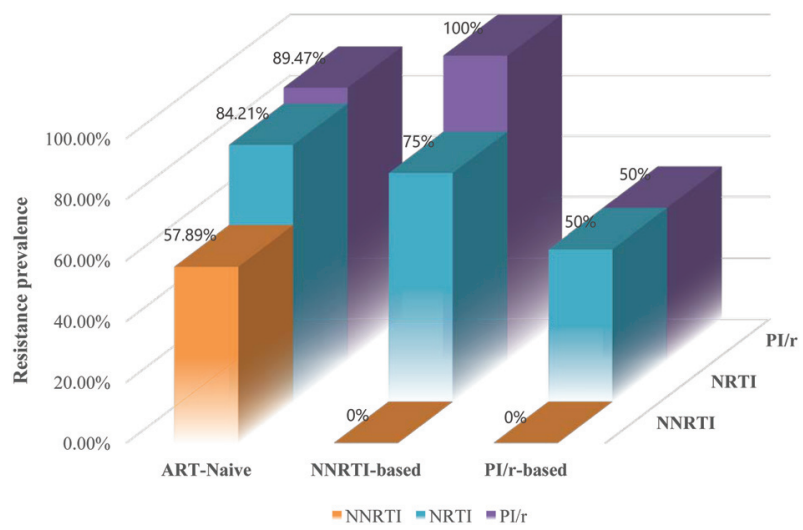

Fig. 3. Level of predictive effectiveness of different ARV drug classes. NNRTI, non-nucleoside reverse transcriptase inhibitors; NRTI, nucleoside reverse transcriptase inhibitors; $\mathrm{Pl}$, protease inhibitor.

The genetic diversity had no major effect on the presence of DRMs $(\mathrm{p}=0.99)$. Table 2 shows the similar distribution of ADR between 02 AG versus non-AG infected participants.

\section{Discussion}

As pediatric HIV infection tends to decrease overtime, follow-
Table 2. Effect of HIV-1 genetic diversity on acquired drug resistance

\begin{tabular}{llll}
\hline Rates of DRMs & CRF02_AG & Non-CRF02_AG & p-value \\
\hline Overall rate & $96.88 \%(31 / 32)$ & $100 \%(12 / 12)$ & 0.53 \\
NNRTI-DRMs & $93.75 \%(30 / 32)$ & $100 \%(12 / 12)$ & 0.52 \\
NRTI-DRMs & $86.63 \%(29 / 32)$ & $91.67 \%(11 / 12)$ & 0.70 \\
PI-DRMs & $15.63 \%(05 / 32)$ & $25 \%(3 / 12)$ & 0.37 \\
\hline
\end{tabular}

CRF, circulating recombinant form; DRMs, drug resistance mutations; HIV-1, human immunodeficiency virus type 1 ; NNRTI, non-nucleoside reverse-transcriptase inhibitors; NRTI, nucleoside reverse-transcriptase inhibitors; PI, protease inhibitors.

ing the success of PMTCT option B + in resource-limited settings (RLS), ${ }^{1}$ efforts should be dedicated for a close monitoring of HIVDR among any infected child. ${ }^{7}$ In this study, we generated findings on HIVDR among children naïve to HAART and those failing ART, as an attempt to contribute for the better management of children who become infected in the era of option B+ in Cameroon, as well as in similar countries in RLS. ${ }^{26,27}$

Among HAART-naïve infants (i.e. all aged below one year), half harbored PDR. This therefore suggests that about half of children infected with HIV may harbor a resistant virus. Though limited in sample size, our findings are in accordance with the recent reports indicating that about five out of ten HIV-infected infants carry resistant viruses. ${ }^{26,28}$ This high rate of PDR is far different from previous reports in the same country $(4.9 \%$ around $2010),{ }^{29}$ and beyond the WHO's threshold for PDR, ${ }^{26}$ a disparity

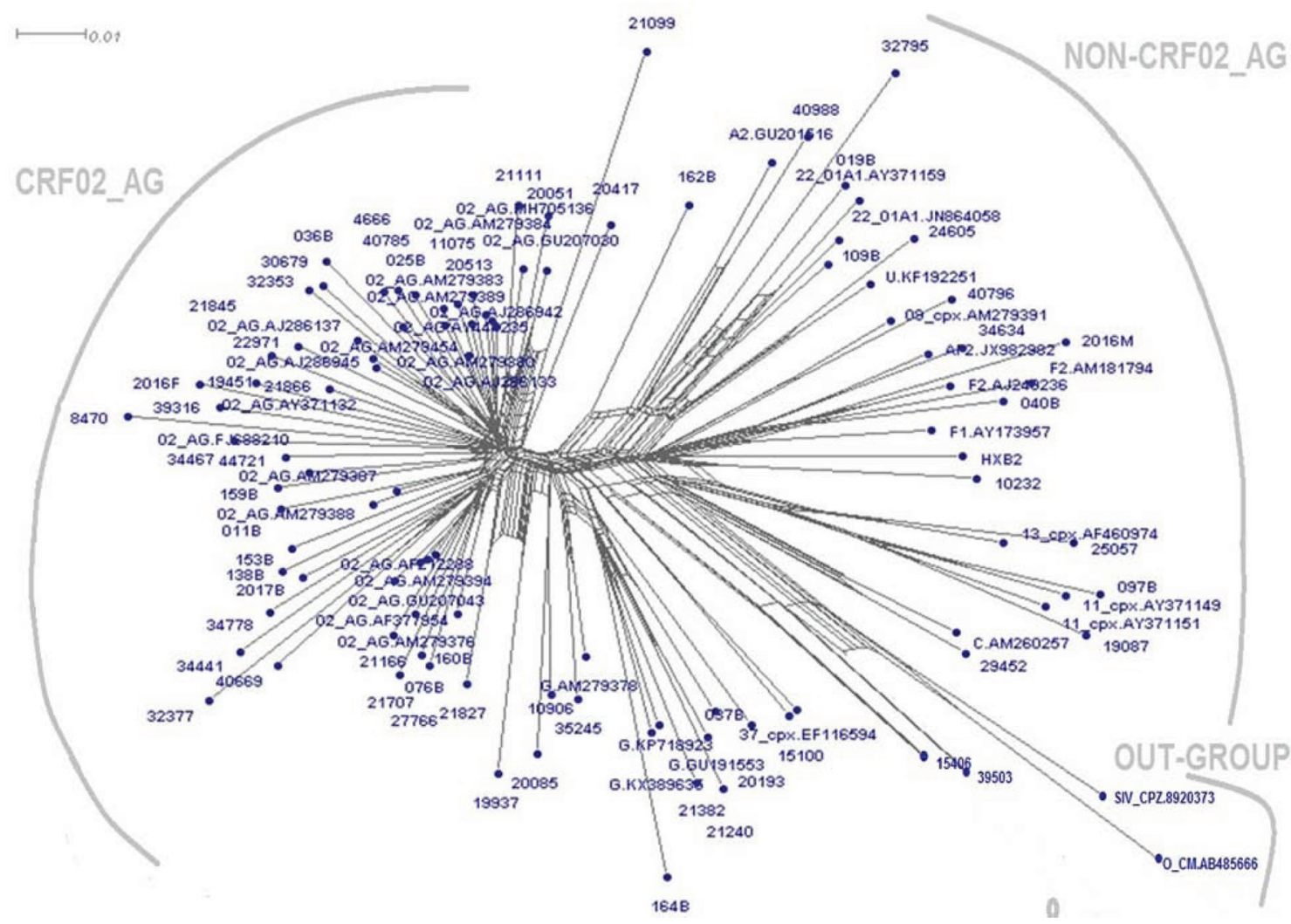

Fig. 4. HIV-1 phylogenetic tree of the 63 perinatally infected participants. The reference sequences were from the Los Alamos Database (https://www.hiv. lanl.gov/components/sequence/HIV/search/search.html); some references have been omitted to enable better visualization. The scale bar represents $1 \%$ genetic distance. CRF, circulating recombinant form. 
mainly attributed to the scale of PMTCT interventions..$^{30}$ Thus, with increasing access to option $\mathrm{B}+$, PDR would be present in more than half of HIV-positive infants, especially with the use of next generation sequencing platforms. ${ }^{31}$ Surprisingly, PDR was also high among children reported to have no exposure to ARV. This is likely due to selection bias attributed to inadequate maternal recall of previous PMTCT interventions (which could have been resolved by measuring for drug concentration), as previously reported around $25 \%$ by Chakanyuka-Musanhu et al., in 2013. ${ }^{30}$ Therefore, without timeous interventions, PDR to NNRTI, NRTI and $\mathrm{PI} / \mathrm{r}$ would further increase overtime among children. ${ }^{32}$ This therefore calls for considerations to either providing HIVDR testing for selecting active ingredients (preferred wherever feasible) or ensuring an effective use of more potent ARV drugs (including raltegravir) as initial therapy for newly diagnosed HIV positive infants. ${ }^{33}$

Among children failing ART, treatment failure occurred about two years after therapeutic initiation (ranging between one to five years for the majority). This confirms the event of early ART failure among children and the need for timeous viral load monitoring, as previously reported in the several settings of the same country. ${ }^{14,22,29}$ This early ART failure associated with high rate of HIVDR should be addressed critically, as this may indicate a rapid selection of DRMs pre-existing as minority variants upon pharmacological pressure, ${ }^{29,31,32}$ and/or compliance challenges as children grow towards adolescence. ${ }^{15,34}$

The high burden of ADR among children failing the first- and second-line regimens reveal almost complete inefficacy of NNRTIs and NRTIs, which are low genetic barrier drugs, contributing to early selection of DRMs among children, especially in the frame of poor compliance to ART regularly reported among children and adolescents. ${ }^{15,29,34}$ Interestingly, dual-class of resistance to NNRTI and NRTI remains very high ( $>80 \%)$ among children failing firstand second-line ART, with very low rate resistance to PI/r. This underscores the benefit of using PI/r-based regimens or other NNRTI-sparing ART combinations as initial or first-line ART among children diagnosed HIV positive. ${ }^{33}$ Of relevance, $50 \%$ of children on second-line have DRMs to PI/r, indicating that about half of children experiencing VF on PI/r-based second line are in need of third-line ART regimens containing integrase strand-transfer inhibitors (dolutegravir, raltegravir) and/or DRV/r. ${ }^{33,35,36}$ However, with about one fifth risk of reduced efficacy of DRV/r $(\sim 19 \%$ in our study) following exposure to the classical PI/r-based regimen, alongside the well documented high risk of inactive RTIs, ${ }^{36}$ close viral load testing and GRT should be strongly recommended prior to switching to third-line ART even in children and adolescents. ${ }^{36-38}$

Despite the broad viral genetic diversity among Cameroonian populations, ${ }^{39,40}$ there was no considerable effect of the major circulating subtype (i.e. CRF02_AG) on emerging DRMs. ${ }^{29,31}$ Therefore, although HIV variability may be associated to viral fitness, disease progression and drug susceptibility, ${ }^{41}$ our findings reveal that subtype disparity might not have a major clinical relevance in the Cameroonian pediatric context at the moment. ${ }^{34,36}$

Our study has some limitations. Firstly, the limited sample size in HAART-naïve children diagnosed HIV-positive in our EID program (due to declining vertical transmission with PMTCT success and treat all implementation) restricted the breath of PDR appraisal and the true effect of PMTCT (due to recall bias). Thus, clinicians should not focus on maternal recall on PMTCT-exposure in guiding pediatric ART regimens. ${ }^{30}$ Furthermore, testing for drug concentration (not performed in our study) for uncertain exposure to PMTCT would help in screening children with potential wild type viruses at ART initiation.

\section{Future directions}

For a better understanding of the impact of PDR in pediatric populations, further investigations will focus on assessing drug resistance patterns in a larger population, considering the selection of mutations according to HIV-1 subtypes/recombinants, maternal ART history and feeding options throughout the PMTCT cascade care. Assessing the effect of infant age on the trends of PDR would also help in timing pediatric ART regimen. Lastly, surveys of acquired HIVDR to newer drugs will help in tailoring future optimal second/third-line pediatric regimens.

\section{Conclusions}

In a nutshell, HIVDR appeared very high among Cameroonian children. The high burden of PDR detected at infancy could be due to scale-up in PMTCT. The early VF ( 24 months of pediatric-ART) was associated to the presence of ADR, with multidrug resistance to RTIs that requires innovative drugs (integrase strand transfer inhibitor, preferably and DRV/r). This advocates for GRT to guide initial ART and to select for optimal ART regimens when switching to subsequent regimens in children. The broad HIV-1 genetic variability does not appear with any potential clinical relevance on the emergence of DRMs.

\section{Acknowledgments}

We are appreciative to our institutional staff (CIRCB) who participated in the enrolment and in sample processing. We are thankful to the children and adolescents, their parents or guardian for their consents. We also acknowledge Ghylaine Bruna Djeunang Dongho, Armand Tiotsia Tsapi and Irenée Domkam for their comments and remarks in the data analysis and interpretation of findings.

\section{Funding}

The present study was supported the funds of the Chantal BIYA International Reference Centre for research on HIV/AIDS prevention and management (CIRCB), under the CIRCB annual budget 2017-2018

\section{Conflict of interest}

The authors have no conflict of interests related to this publication.

\section{Author contributions}

Conceived the study and designed the experiment (BD, JF, ESN), acquired the data (BD, JF, ESN, DT, GT), analyzed and interpreted the data (BD, JF, ESN, LCMM, NK, SMS, SCB, AEN, MSS, $\mathrm{CN}, \mathrm{PNK}, \mathrm{FN}, \mathrm{VC}, \mathrm{CFP}, \mathrm{AN})$; drafting of the manuscript (BD, JF, ESN), critical revision of the manuscript for important intellectual content (DT, MSS, GT, GB, LCMM), study supervision (CN, 
PNK, FN, VC, CFP, AN); approved the final version submitted (all the authors).

\section{References}

[1] UNICEF. Option Band B+:Keyconsiderationsforcountriesto implement an equity-focused approach. Geneva: UNICEF; 2012. Available from: https://www.unicef.org/aids/files/hiv_Key_considerations_options_ B.pdf.

[2] Boerma RS, Boender TS, Bussink AP, Calis JC, Bertagnolio S, Rinke de Wit TF, et al. Suboptimal viral suppression rates among HIV-infected children in low- and middle-income countries: A Meta-analysis. Clin Infect Dis 2016;63(12):1645-1654. doi:10.1093/cid/ciw645.

[3] AC Ubesie. Pediatric HIV/AIDS in sub-Saharan Africa: emerging issues and way forward. Afr Health Sci 2012;12(3):297-304. doi:10.4314/ ahs.v12i3.8.

[4] UNAIDS. Children and HIV: Fact sheet. Geneva: Joint United Nations Programme on HIV/AIDS; 2016. Available from: https://www.unaids. org/sites/default/files/media_asset/FactSheet_Children_en.pdf.

[5] UNAIDS. Global AIDS updates. Geneva: Joint United Nations Programme on HIV/AIDS; 2016. Available from: https://www.unaids.org/ sites/default/files/media asset/global-AIDS-update-2016 en.pdf.

[6] UNAIDS. UNAIDS 2016 estimates (Cameroon). Genève: UNAIDS. 2016:60-61.

[7] Inzaule SC, Hamers RL, Calis J, Boerma R, Sigaloff K, Zeh C, et al. When prevention of mother-to-child HIV transmission fails: preventing pretreatment drug resistance in African children. AIDS 2018;32(2):143147. doi:10.1097/QAD.0000000000001696.

[8] Masson D, Hiffler L. Guide de prise en charge de l'infection à VIH chez l'enfant et l'adolescent. 2nd edition. Programme GRANDIR. 2014:5492.

[9] McNairy ML, Lamb MR, Carter RJ, Fayorsey R, Tene G, Mutabazi V, et al. Retention of HIV-infected children on antiretroviral treatment in HIV care and treatment programs in Kenya, Mozambique, Rwanda, and Tanzania. J Acquir Immune Defic Syndr 2013;62(3):e70-e81. doi:10.1097/QAl.0b013e318278bcb0.

[10] World Health Organization. Global action plan on HIV drug resistance 2017-2021: 2018 progress report. 2018. Available from: https://apps. who.int/iris/bitstream/handle/10665/273049/WHO-CDS-HIV-18.12eng.pdf.

[11] World Health Organization. Consolidated guidelines on the use of antiretroviral drugs for treating and preventing HIV infection: recommendations for a public health approach, 2nd edition. 2016. Available from: https://apps.who.int/iris/handle/10665/208825.

[12] UNAIDS. Start Free, Stay Free, AIDS Free - A super-fast-track framework for ending AIDS among children, adolescents and young women by 2020. Bangkok: HIV and AIDS Data Hub for Asia Pacific; 2016. Available from: https://aidsdatahub.org/start-free-stay-freeaids-free-super-fast-track-framework-ending-aids-among-childrenadolescents-and.

[13] Davies MA, Pinto J, Bras M. Getting to $90-90-90$ in paediatric HIV: what is needed? J Int AIDS Soc 2015;18(7 Suppl 6):20770. doi:10.7448/IAS.18.7.20770.

[14] Njom Nlend AE, Motaze AN, Ndiang ST, Fokam J. Predictors of virologic failure on first-line antiretroviral therapy among children in a referral pediatric center in Cameroon. Pediatr Infect Dis J 2017;36(11):1067-1072. doi:10.1097/INF.0000000000001672.

[15] Fokam J, Billong SC, Jogue F, Moyo Tetang Ndiang S, Nga Motaze $\mathrm{AC}$, Paul KN, et al. Immuno-virological response and associated factors amongst HIV-1 vertically infected adolescents in YaoundéCameroon. PLoS One 2017;12(11):e0187566. doi:10.1371/journal. pone. 0187566 .

[16] Boyer S, Eboko F, Camara M, Abe C, Nguini ME, Koulla-Shiro S, et al. Scaling up access to antiretroviral treatment for HIV infection: the impact of decentralization of healthcare delivery in Cameroon. AIDS 2010;24 Suppl 1:S5-15. doi:10.1097/01.aids.0000366078.45451.46.

[17] MINSANTE. Guide pour la prise en charge des enfants exposes et infectes par le VIH/SIDA - version simplifiee. National Pediatrics ARV guidelines. Yaoundé-Cameroun. 2012:3-78.
[18] MINSANTE. Plan d'accélération de la thérapie ARV au Cameroun 2016-2018. Principaux résultats de la thérapie antirétrovirale. Yaoundé-Cameroun: Comité national de lutte contre le VIH/sida. 2015:510.

[19] MINSANTE. Directives nationales de prévention et de prise en charge du VIH/SIDA. Yaoundé-Cameroun. 2015:12-65.

[20] Paredes R, Marconi VC, Lockman S, Abrams EJ, Kuhn L. Impact of antiretroviral drugs in pregnant women and their children in Africa: HIV resistance and treatment outcomes. J Infect Dis 2013;207 Suppl 2:S93-100. doi:10.1093/infdis/jit110.

[21] Ton Q, Frenkel L. HIV drug resistance in mothers and infants following use of antiretrovirals to prevent mother-to-child transmission. Curr HIV Res 2013;11(2):126-136. doi:10.2174/1570162X11311020005.

[22] Penda Cl, Bebey FS, Mangamba DK, Moukoko EC, Ngwa V, Makouet $\mathrm{N}$, et al. Treatment failure in children infected with HIV in routine follow-up in a resource-limited setting in Cameroon. Pan Afr Med J 2013;15:80. doi:10.11604/pamj.2013.15.80.2754.

[23] UNICEF. Children and AIDS: Statistical update. 2017. Available from: https://data.unicef.org/resources/children-aids-statistical-update/.

[24] Fokam J, Salpini R, Santoro MM, Cento V, D’Arrigo R, Gori C, et al. Performance evaluation of an in-house human immunodeficiency virus type-1 protease-reverse transcriptase genotyping assay in Cameroon. Arch Virol 2011;156(7):1235-1243. doi:10.1007/s00705-0110982-3.

[25] Martin DP, Murrell B, Golden M, Khoosal A, Muhire B. RDP4: detection and analysis of recombination patterns in virus genomes. Virus Evol 2015;1(1):vev003. doi:10.1093/ve/vev003.

[26] World Health Organization. HIV drug resistance report 2017. Available from: https://www.who.int/hiv/pub/drugresistance/hivdr-report-2017/en/.

[27] Bertagnolio S, Penazzato M, Jordan MR, Persaud D, Mofenson LM, Bennett DE, et al. World Health Organization generic protocol to assess drug-resistant HIV among children $<18$ months of age and newly diagnosed with HIV in resource-limited countries. Clin Infect Dis 2012;54 Suppl 4:S254-S260. doi:10.1093/cid/cis003.

[28] Kuhn L, Hunt G, Technau KG, Coovadia A, Ledwaba J, Pickerill S, et al. Drug resistance among newly diagnosed HIV-infected children in the era of more efficacious antiretroviral prophylaxis. AIDS 2014;28(11):1673-1678. doi:10.1097/QAD.0000000000000261.

[29] Fokam J, Salpini R, Santoro MM, Cento V, Perno CF, Colizzi V, et al. Drug resistance among drug-naive and first-line antiretroviral treatment-failing children in Cameroon. Pediatr Infect Dis J 2011;30(12):1062-1068. doi:10.1097/INF.0b013e31822db54c.

[30] Chakanyuka-Musanhu C, Penazzato M, Apollo T, Dzangare J, MtapuriZinyowera S, Mugurungi O, et al. World Health Organization HIV drug resistance surveillance in children less than 18 months newly diagnosed with HIV in Zimbabwe. Kuala Lumpur, Malaysia: International AIDS Society 2013. 2013.

[31] Fokam J, Bellocchi MC, Armenia D, Nanfack AJ, Carioti L, Continenza F, et al. Next-generation sequencing provides an added value in determining drug resistance and viral tropism in Cameroonian HIV-1 vertically infected children. Medicine 2018;97(13):e0176. doi:10.1097/ MD.0000000000010176.

[32] Boerma RS, Sigaloff KC, Akanmu AS, Inzaule S, Boele van Hensbroek $\mathrm{M}$, Rinke de Wit TF, et al. Alarming increase in pretreatment HIV drug resistance in children living in sub-Saharan Africa: a systematic review and meta-analysis. J Antimicrob Chemother 2017;72(2):365371. doi:10.1093/jac/dkw463.

[33] World Health Organization. Updated recommendations on first-line and second-line antiretroviral regimens and post-exposure prophylaxis and recommendations on early infant diagnosis of HIV. $2018 \mathrm{ed}$. Geneva: World Health Organization. 2018:1-16.

[34] Boerma RS, Bunupuradah T, Dow D, Fokam J, Kariminia A, Lehman $\mathrm{D}$, et al. Multicentre analysis of second-line antiretroviral treatment in HIV-infected children: adolescents at high risk of failure. J Int AIDS Soc 2017;20(1):21930. doi:10.7448/IAS.20.1.21930.

[35] Bruzzese E, Lo Vecchio A, Smarrazzo A, Tambaro O, Palmiero G, Bonadies $G$, et al. Dolutegravir-based anti-retroviral therapy is effective and safe in HIV-infected paediatric patients. Ital J Pediatr 2018;44(1):37. doi:10.1186/s13052-018-0469-x.

[36] Njom Nlend AE, Guessong CO, Nga Motaze AC, Soffo C, Koki Ndombo 
PO, Tsambang L, et al. Outcomes of protease inhibitor-based antiretroviral therapy amongst children and associated-factors in Yaoundé, Cameroon. PLoS One 2019;14(3):e0213900. doi:10.1371/journal. pone. 0213900 .

[37] Raizes E, Hader S, Birx D. Expansion of Viral Load Testing and the Potential Impact on HIV Drug Resistance. J Infect Dis 2017;216(suppl_9):S805-S807. doi:10.1093/infdis/jix432.

[38] Takou D, Fokam J, Teto G, Santoro MM, Ceccherini-Silberstein F, Nanfack AJ, et al. HIV-1 drug resistance testing is essential for heavily-treated patients switching from first- to second-line regimens in resource-limited settings: evidence from routine clinical practice in
Cameroon. BMC Infect Dis 2019;19(1):246. doi:10.1186/s12879-0193871-0.

[39] Vallari A, Bodelle P, Ngansop C, Makamche F, Ndembi N, Mbanya D, et al. Four new HIV-1 group $\mathrm{N}$ isolates from Cameroon: prevalence continues to be low. AIDS Res Hum Retroviruses 2010;26(1):109115. doi:10.1089/aid.2009.0178.

[40] Vallari A, Holzmayer V, Harris B, Yamaguchi J, Ngansop C, Makamche $\mathrm{F}$, et al. Confirmation of putative HIV-1 group P in Cameroon. J Virol 2011;85(3):1403-1407. doi:10.1128/JVI.02005-10.

[41] Daar ES. HIV-1 virulence, fitness and replication capacity. Therapy 2005;2(1):131-140. 